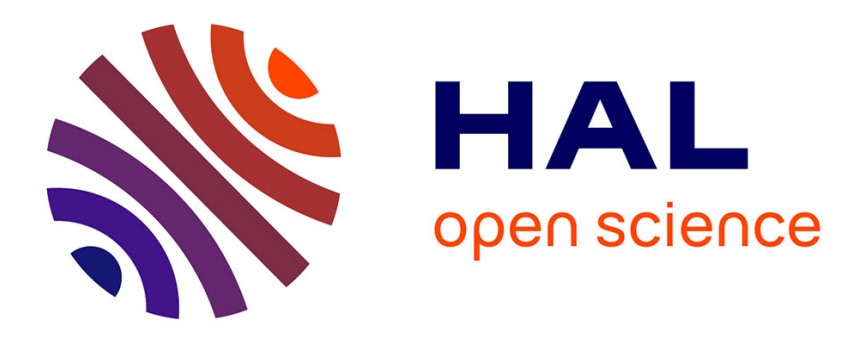

\title{
Supermultiplet mass predictions for exotic nuclei
}

V. Gillet, A. Klein

\section{To cite this version:}

V. Gillet, A. Klein. Supermultiplet mass predictions for exotic nuclei. Journal de Physique Lettres, 1979, 40 (15), pp.359-361. 10.1051/jphyslet:019790040015035900 . jpa-00231644

\section{HAL Id: jpa-00231644 https://hal.science/jpa-00231644}

Submitted on 1 Jan 1979

HAL is a multi-disciplinary open access archive for the deposit and dissemination of scientific research documents, whether they are published or not. The documents may come from teaching and research institutions in France or abroad, or from public or private research centers.
L'archive ouverte pluridisciplinaire HAL, est destinée au dépôt et à la diffusion de documents scientifiques de niveau recherche, publiés ou non, émanant des établissements d'enseignement et de recherche français ou étrangers, des laboratoires publics ou privés. 


\title{
LE JOURNAL DE PHYSIQUE-LETTRES
}

\section{Supermultiplet mass predictions for exotic nuclei}

\author{
V. Gillet and A. Klein \\ Département de Physique Nucléaire, C.E.N. Saclay, B.P. 2, 91190 Gif sur Yvette, France
}

(Reçu le 18 mai 1979, accepté le 14 juin 1979)

Résumé. - Le remplacement des termes d'énergie de symétrie et d'appariement par l'opérateur de Casimir C2 de SU4 améliore fortement les prédictions de masse pour les isotopes déficients en neutrons des césium et rubidium.

Abstract. - Replacement of the symmetry and pairing energy terms by the Casimir operator C2 of SU4 improves the mass predictions for neutron deficient caesium and rubidium isotopes.

Nuclear masses near the stability valley have been shown to be well described by an SU4 mass formula [1]. A more recent and detailed calculation by $\mathbf{M}$. Cauvin et al. [2] confirms this result. We report here on the application of the model to nuclei very far from the stability line, using the recent ISOLDE data on caesium and rubidium isotopes [3].

Franzini and Radicatti [4], Burdet et al. [5] have proposed various mass relationships for testing the SU4 character of the effective nuclear Hamiltonian in the ground states of medium and heavy nuclei. The agreements which they obtained were surprisingly good in view of the strong spin-orbit shell model potential. However the meaning of such results was questioned, for example by Zeldes and Taraboulos [6] who noted that they were based on ratios of mass differences related to the curvature of the isobar mass parabolas : thus for nuclei situated outside the stability region, where the parabola curvatures are small, the tests were not very specific of a particular model.

A more straightforward approach consists of course in comparing directly the masses parametrized in terms of the Casimir operators of SU4 with the experimental masses. Thus it was shown that a simple mass formula where the C2 Casimir operator is substituted for the ordinary symmetry energy and pairing energy accounts for the experimental masses as well as or better than the Weizsäcker formula [1, 2].

A justification for a possible effective SU4 Hamiltonian symmetry in medium and heavy nucleus ground states can be given within the quartet model [7]. The strong alignment effect of proton-neutron correlations dominates over the pairing antialignment.
This dominance leads to the preferential filling in the ground state of configurations made up of components like $|j m\rangle_{\mathbf{p}}|j m\rangle_{\mathbf{n}}|j-m\rangle_{\mathbf{p}}|j-m\rangle_{\mathbf{n}}$. For such configurations the terms $\sigma_{\mathrm{i}}, \sigma_{\mathrm{j}}$ and $\tau_{\mathrm{i}}, \tau_{\mathrm{j}}$ of the central force yield contributions proportional to $A$, which simply add up to the volume term, and to $T(T+1)$ which are partially contained in $\mathrm{C} 2$, the second-order SU4 Casimir operator.

As a more stringent test of the model it is of interest to investigate the case of very exotic nuclei, either with large neutron excess (which should deviate from the SU4 picture because of the dominance of pairing correlations in the very large valence neutron system) or with large neutron deficiency.

The recent series of rubidium and caesium isotopes obtained at CERN by the ISOLDE collaboration [3] offer an interesting sample for carrying out this SU4 test. The two models which are compared are the usual Weizsäcker formula with pairing $(\lambda(N, Z)=0$ for $A$ odd, $\lambda(N, Z)=+1$ for odd-odd and $\lambda(N, Z)=-1$ for even-even nuclei)

$$
\begin{array}{r}
B=\alpha A+\beta A^{2 / 3}+0.72 \frac{Z^{2}}{A^{1 / 3}}+\gamma 4 T^{2} A^{-1}+ \\
+\delta \lambda(N, Z) A^{-0.5}
\end{array}
$$

and the supermultiplet expression limited to the ;(C2) $A^{\eta}$ term representing two-body correlations

$$
\begin{aligned}
B=\alpha^{\prime} A+\beta^{\prime} & A^{2 / 3}+0.72 \frac{Z^{2}}{A^{1 / 3}}+ \\
& +\gamma\left(T(T+4)+\frac{3}{2} \lambda(N, Z)\right) A^{\eta}
\end{aligned}
$$


We note that the amplitude of the periodic $\lambda(N, Z)$ factor is not a free parameter anymore in the latter expression.

In each proton-neutron major shell region $i$, the shell effects are simulated by a five-parameter expansion

$$
E_{\mathrm{i}}=a_{\mathrm{i}}+b_{\mathrm{i}} N+c_{\mathrm{i}} N^{2}+d_{\mathrm{i}} Z+e_{\mathrm{i}} Z^{2} .
$$

In a first step the shell effect parameters, eq. (3), and the mass formula parameters, eq. (1) or eq. (2), are determined by a best fit in each proton-neutron major shell region i. We extract from reference [2] a partial list of results for the root mean square of the difference between the theoretical and experimental masses $(\overline{\Delta M})$ in the four regions $i$ which are relevant for getting the shell model parameters used for the rubidium and caesium isotopes, see table $I$, step 1 . In a second step the shell energy parameters, eq. (3), are kept constant at their values of step one and the 4 other mass parameters, eq. (1) or eq. (2), are varied to give a best fit for all known 1628 nuclides as of 1975 [8]. The results ( $\overline{\Delta M}$ and parameter values) are also given in table I, step 2. The mass formula parameters thus obtained are then applied without any further variation to the prediction of exotic nuclei.

The results of the application of the model to the exotic rubidium and caesium masses are given in figures 1 and 2. The experimental masses are taken from the ISOLDE work [3]. There are 26 rubidium isotopes $(Z=37 ; 37 \leqslant N \leqslant 62)$ and 30 caesium isotopes $(Z=55 ; 62 \leqslant N \leqslant 93)$. The errors $B$ (theor.) $-B$ (exp.) are given in $\mathrm{keV}$ in the figures.

It is seen from the results of figures 1 and 2 that the supermultiplet mass formula yields a satisfactory

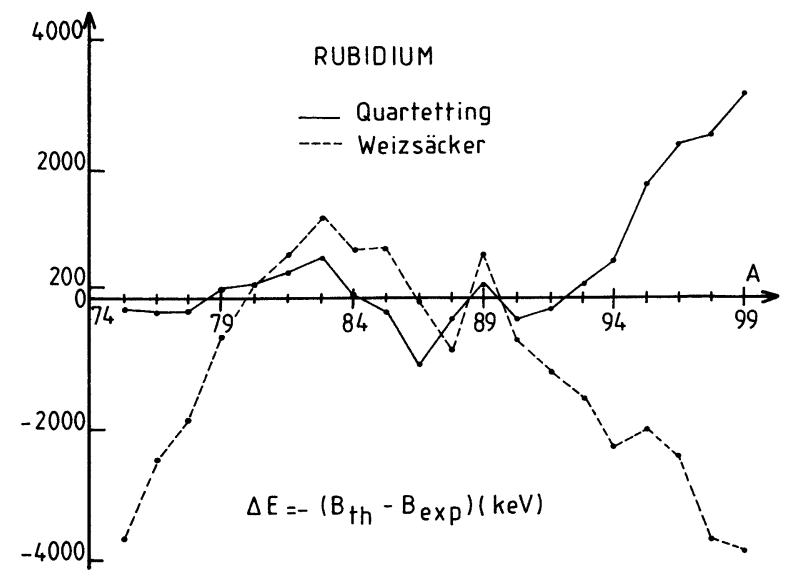

Fig. 1. - Prediction of rubidium isotope masses.

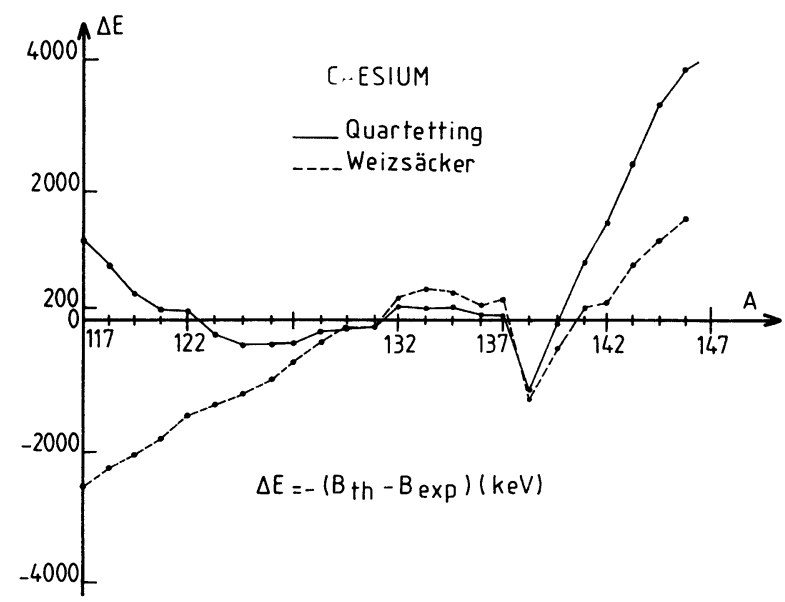

Fig. 2. - Prediction of caesium isotope masses.

Table I. - Parameter determination in formulas (1), (2), (3)

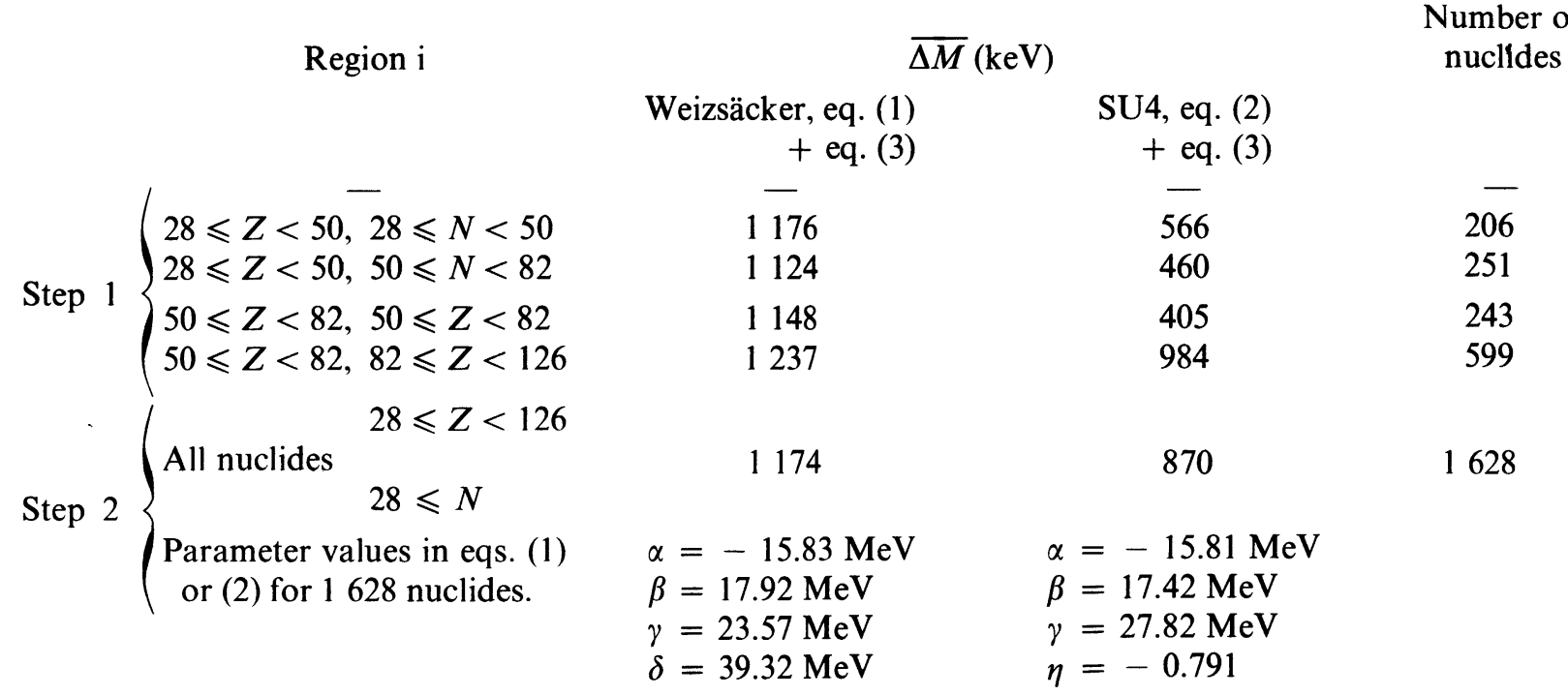

From M. Cauvin et al. [2]. 
agreement not only near the minimum of the isobaric mass parabolas, as found in refs. [1] and [2], but also far away on the neutron deficient side of these parabolas. For the lowest $|N-Z|$ values the SU4 form appears in fact much superior to the Weizsäcker formula or at least as successful as more refined mass formulas containing a greater number of parameters [3]. The origin of the improvement over the Weizsäcker form is the linear dependence in $T$ contained in $\mathrm{C} 2$, eq. (2). We note however that the errors are rapidly increasing for nuclei with increasing neutron excesses. These results can be interpreted in the framework of the quartetting model [7], according which protonneutron alignment dominates over the pairing effect in doubly open (protons and neutrons) shell nuclei situated near the bottom of the stability valley or on the neutron deficient side. It is only for the largest neutron excesses that the $T=1$ pairing effect takes over [9].

Acknowledgments. - The authors acknowledge gratefully the help of Monique Cauvin for the use of her computing code.

\section{References}

[1] Danos, M. and Gillet, V., Proceedings of the Tokyo Conference on Nuclear Physics (1977).

[2] Cauvin, M., Danos, M. and Gillet, V., to be submitted to Nucl. Phys.

[3] Epherre, M., Audi, G., Thibault, C., Klapisch, R., Huber, G., Touchard, F., Wollnick, H. and Isolde Col., LRB report 78-09 (CNSM, Orsay) and in print Phys. Rev. C (April 1979). The authors are grateful to R. Klapisch and $\mathbf{M}$. Epherre for communication of the Isolde collaboration results prior to publication.
[4] Franzini, P. and Radicatti, L. A., Phys. Lett. 6 (1963) 322.

[5] Burdet, J., Maguin, C. and Partensky, A., Nuovo Cimento 54B (1968) 1.

[6] Zeldes, N. and Taraboulos, A., First Oaxtepec Symposium on Nuclear Physics (Oaxtepec, 1978).

[7] Danos, M. and Gillet, V., Z. Phys. 249 (1972) 204

[8] Wapstra, A. H. and Bos, K., At. Data Nucl. Data Tables 19 (1977) 175.

[9] Gillet, V. and Raynal, J., Nucl. Phys. A 122 (1968) 193. 